\title{
Gute Freunde: Alkohol und Allergie
}

\begin{abstract}
Die in den letzten Jahrzehnten zunehmende Sensibilisierung speziell gegen Aeroallergene, aber auch die Zunahme allergischer Erkrankungen insgesamt wird mit Auswirkungen der westlichen Überflussgesellschaft in Zusammenhang gebracht. Einer dieser „Lifestyle“-Faktoren könnte der Alkoholkonsum sein.
\end{abstract}

\begin{abstract}
C $s$ ist bekannt, dass das Trinken von
Alkohol allergische Reaktionen triggern kann, zu einer Erhöhung des Gesamt-IgE führt und Veränderungen im Zytokinprofil induziert. Alkoholgenuss während der Schwangerschaft führt zu erhöhten IgE-Spiegeln im Nabelschnurblut.

Dänische Epidemiologen dokumentierten über zwei Jahre hinweg kontinuierlich den per Fragebogen ermittelten Alkoholkonsum von 3.317 Frauen und Männern zwischen 30 und 60 Jahren. Der Alkoholkonsum pro Woche wurde gesondert für Bier, Wein und hochprozentige Getränke erfasst und unterteilt in geringen, mittelstar-

Ein gutes Glas Rotwein mag kardioprotektiv wirken, vor Allergien schützt es nicht.
\end{abstract}

ken und sehr starken Konsum. Die Vertrauenswürdigkeit der Angaben zum Alkoholkonsum wurde durch Bestimmung der

Gamma-GT überprüft. Weiter wurden Serumproben der Studienteilnehmer mittels des automatisierten Multi-Screening-Systems ADVIA Centaur $^{\circledR}$ auf spezifisches IgE gegen die 16 wichtigsten Aeroallergene analysiert. Um einen Zusammenhang zwischen Alkoholgenuss und Sensibilisierung herstellen zu können, wurden Regressionsanalysen vorgenommen. $\mathrm{Da}$ bei wurden die Einflussgrössen Alter, Geschlecht, Tabakkonsum, Bildungsstand und

Body-Mass-Index statistisch berücksichtigt.

Es zeigte sich eindeutig der Trend, dass mit zunehmendem Alkoholkonsum die Sensibilisierung gegen Aeroallergene zunahm - je stärker der Alkoholkonsum desto stärker die Sensibilisierung. Statistisch eindeutig gesichert war dieser $\mathrm{Zu}-$ sammenhang für die Gruppe mit sehr hohem Alkoholonsum, der definiert war als 15-20 Drinks (180-240 g Alkohol) pro Woche. Die Art des Alkohols spielt dabei keine Rolle, was auf die alleinige Einflussnahme des Alkohols beim Sensibilisierungsprozess hindeutet und die Rolle von biogenen Aminen oder Aromaund Konservierungsstoffen relativiert.

\section{Fazit}

Die Ergebnisse dieser dänischen Untersuchung dürften auf deutsche Verhältnisse übertragbar sein. Demnach ist allen Allergiekranken, insbesondere Asthmapatienten zu empfehlen, ihren Alkoholgenuss möglichst zu reduzieren, besser noch, abstinent zu leben.

Dr. Dieter Bruchhausen, Wupperta

\section{Literatur}

Linneberg A, Hertzum I, Husemoen LL, Johansen $\mathrm{N}$, Jorgensen $\mathrm{T}$. Association between alcohol consumption and aeroallergen sensitization in Danish adults. Clin Exp Allergy 2006; 36: 714-21

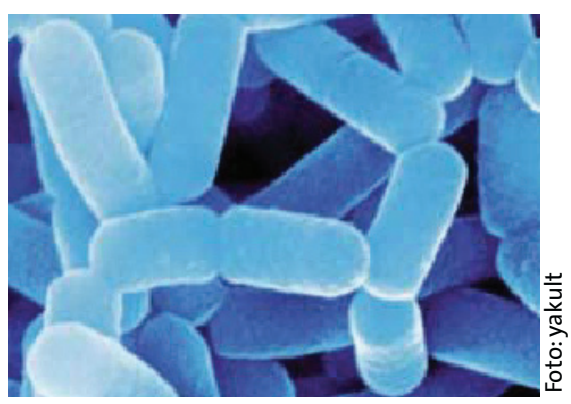

ahlreiche Arbeiten belegen, dass frühkindliche Infektionskrankheiten das Allergierisiko reduzieren, auf der anderen Seite die Behandlung von Infektionen mit Antibiotika das Allergierisiko erhöht. Welche Mikroorganismen hinter diesem allergieprotektiven Effekt stecken, ist allerdings unklar.

Die individuelle Zusammensetzung der mikrobiellen Darmflora des gesunden Erwachsenen bleibt lebenslang weitgehend konstant, wohingegen sich die des
Säuglings physiologischerweise von Geburt an, dann über die Abstillung hinaus bis zum zweiten Lebensjahr ständig ändert. In Anbetracht der wichtigen Rolle, welche die mikrobielle Besiedlung des Darms für die Entwicklung des Immunsystems spielt, wird verständlich, dass aus einer Störung der Darmflora auch Störungen im Immunsystem resultieren können. So gehen quantitative und qualitative Veränderungen der frühkindlichen Bifidusflora allergischen Erkrankungen
Meist handelt es sich bei Probiotoka um Stämme von Bifidobacterium oder Lactobacillus (hier: Lactobacillus casei Shirota). Sie werden aus der Darmflora Gesunder oder aus vergärenden Milchprodukten gewonnen. Seltener werden auch Saccharomyces-Spezies verwendet.

voraus und können diese bis ins späte Kindesalter begleiten. Dies trifft insbesondere für die sich oft bereits im Kleinkindalter manifestierende Neurodermitis zu. 